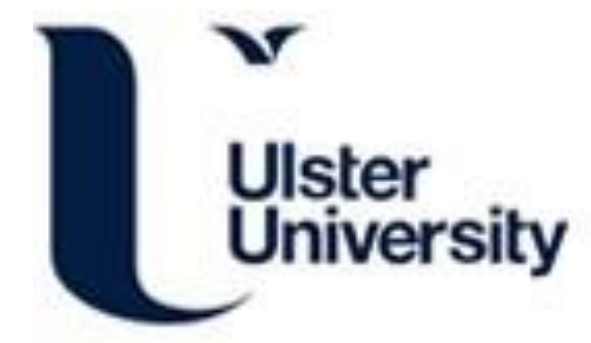

\title{
A connectivity mapping approach predicted acetylsalicylic acid (aspirin) to induce osteo/odontogenic differentiation of dental pulp cells
}

Rankin, R., Lundy, F. T., Schock, B. C., Zhang, S. D., Al-Natour, B., About, I., Irwin, C., Linden, G. J., \& ElKarim, I. A. (2020). A connectivity mapping approach predicted acetylsalicylic acid (aspirin) to induce osteo/odontogenic differentiation of dental pulp cells. International Endodontic Journal, 53(6), 834-845. https://doi.org/10.1111/iej.13281

Link to publication record in Ulster University Research Portal

\section{Published in:}

International Endodontic Journal

Publication Status:

Published (in print/issue): 30/06/2020

DOI:

10.1111/iej.13281

\section{Document Version}

Author Accepted version

\section{General rights}

Copyright for the publications made accessible via Ulster University's Research Portal is retained by the author(s) and / or other copyright owners and it is a condition of accessing these publications that users recognise and abide by the legal requirements associated with these rights.

\section{Take down policy}

The Research Portal is Ulster University's institutional repository that provides access to Ulster's research outputs. Every effort has been made to ensure that content in the Research Portal does not infringe any person's rights, or applicable UK laws. If you discover content in the Research Portal that you believe breaches copyright or violates any law, please contact pure-support@ulster.ac.uk. 
DR. IKHLAS EL KARIM (Orcid ID : 0000-0002-5314-7378)

Article type : Original Scientific Article

A connectivity mapping approach predicted acetylsalicylic acid (aspirin) to induce osteo/odontogenic differentiation of dental pulp cells

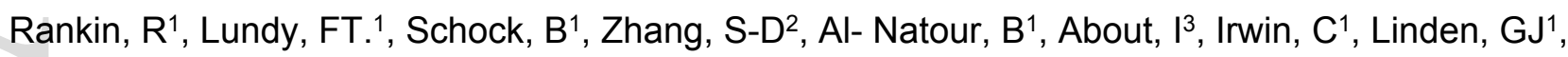
El-Karim, I A 1 .

${ }^{1}$ School of Medicine, Dentistry and Biomedical Sciences, Queen's University Belfast, Belfast, UK ${ }^{2}$ School of Biomedical Sciences, University of Ulster, Derry Londonderry, UK ${ }^{3}$ Faculté d'Odontologie, UMR 7287 CNRS \& Université d'Aix-Marseille, Marseille, France

Running title: Aspirin and DPSC differentiation

Key words: Aspirin, connectivity mapping, odontogenic differentiation, pulp capping, pulpitis

\section{Corresponding author: Dr Ikhlas EI Karim}

Centre for Experimental Medicine, School of Medicine, Dentistry and Biomedical Sciences, Queen's University Belfast, The Wellcome-Wolfson Building, 97 Lisburn Road, Belfast, BT9 7AE, Northern Ireland, United Kingdom

Tel: +442890976026

Email: i.elkarim@qub.ac.uk

This article has been accepted for publication and undergone full peer review but has not been through the copyediting, typesetting, pagination and proofreading process, which may lead to differences between this version and the Version of Record. Please cite this article as doi: $\underline{10.1111 / \text { IEJ.13281 }}$

This article is protected by copyright. All rights reserved 


\section{Abstract}

Aim To use the connectivity map, a bioinformatics approach, to identify compounds that could induce odontogenic differentiation of dental pulp cells (DPCs) and to experimentally validate this effect. A subsidiary aim was to investigate the anti-inflammatory effect of any identified compound.

Methodology The Gene Expression Omnibus (GEO) database was searched for microarray datasets assessing odontogenic differentiation of human DPCs. An odontogenic gene expression signature was generated by differential expression analysis. The statistical significant connectivity map (ssCMap) method was used to identify compounds with a highly correlating gene expression pattern. DPCs were treated with the compound identified and osteo/odontogenic differentiation was assessed by alizarin red staining, alkaline phosphatase activity and expression of osteo/odontogenic genes ALPL, RUNX2, COL1A1, DSPP, DMP1 and SPP1 by RT-PCR. The anti-inflammatory effect of the compound was assessed using an ex vivo pulpitis model and cytokine levels were measured with multiplex assay. Means were compared using the $t$ - test or ANOVA followed by a Bonferroni post hoc -test with the level of significance set at $p \leq 0.05$.

Results The GEO database search identified a specific gene expression signature for osteo/odontogenic differentiation. Analysis using the ssCMap found that acetylsalicylic acid (ASA)/aspirin was the drug with the strongest correlation to that gene signature. The treatment of DPCs with $0.05 \mathrm{mM}$ ASA showed increased alkaline phosphatase activity $(p<0.001)$, mineralisation $(p<0.05)$, and increased the expression of the osteo/odontogenic genes, DMP1 and DSPP $(p<0.05)$. Low concentration $(0.05 \mathrm{mM})$ ASA reduced inflammatory cytokines IL-6 $(p<0.001)$, CCL21 $(P<0.05)$ and MMP-9 $(p<0.05)$ in an ex vivo pulpitis model.

Conclusions Connectivity mapping, a web based informatics method, was successfully used to identify aspirin as a candidate drug that could modulate the differentiation of DPCs. Aspirin was shown to induce odontogenic differentiation in DPCs in vitro and this, together with its antiinflammatory effects, makes it a potential candidate for vital pulp therapies.

This article is protected by copyright. All rights reserved 


\section{Introduction}

The dental pulp is populated with multipotent adult stem cells that confer capacity to regenerate the dentine-pulp complex following damage caused by caries and trauma (Sloan \& Smith 2007). Vital pulp therapies including direct and indirect pulp capping offer opportunities for repair provided an optimal environment is created for activation, recruitment and differentiation of dental pulp stem cells into odontoblast-like cells to produce tertiary dentine. Traditionally, calcium hydroxide has been the material of choice for pulp capping but clinical long term outcomes have been controversial (Murray \& García-Godoy 2006). The introduction of calcium silicate cements has resulted in improved clinical outcomes, compared to calcium hydroxide (Li et al. 2015), but there is still an unmet need for biologically based therapies for dentine repair and regeneration (Kearney et al. 2018).

The improved understanding of the biological processes associated with pulp-dentine regeneration facilitated and informed research leading to the discovery of biologically based pulp therapies. In this regard the use of bioactive molecules, such as TGF- $\beta$, was shown to enhance the regenerative capacity of stem cells and subsequent dentine regeneration (Goldberg et al. 2001, Shrestha \& Kishen 2017). Other approaches that utilised biomolecules known to induce osteogenic effects, demonstrated successful odontogenic differentiation of dental pulp cells (DPCs) (Phung et al. 2017). In addition pharmacological targeting of the $\mathrm{Wnt} / \beta$ catenin signalling pathway, which is known to have a role in dentine repair, demonstrated dentine formation in vivo (Neves et al. 2017).

Bioinformatics provides a novel approach to identify compounds or molecules that could induce dentine formation, based on their biological functions. Gene expression connectivity mapping (CMap) is an innovative web-based technique to establish connections via gene expression profiles/signatures (www.broadinstitute.org/cmap/). The CMap uses a reference database of gene expression profiles representing a series of structured microarray experiments in cell lines to various compounds or drugs. The basic concept of the CMap is to interrogate the reference database with the specific gene expression signature of a disease or a biological condition. Theoretically, these genes may be important for the induction of the phenotype of interest, therefore similar alteration of gene expression by any compound or drug identified would be predicted to induce the same phenotype. One major application of connectivity mapping is to identify potential small molecules able to regulate the expression of a small number of genes (Lamb et al. 2006, Lamb, 2007).

CMap involves the use of a pattern-matching algorithm with a high level of resolution and specificity, however, it has limitations. The method has been further developed into the 'statistically significant connectivity map' (ssCMap) to strengthen reliability by using statistical 
means to control for false connections between gene signature and reference profiles (Zhang \& Gant 2008, 2009, Musa et al. 2017). The ssCMap has been successfully applied to phenotypic targeting and predicting effective drugs for several diseases (Ramsey et al. 2013, Malcomson et al. 2016).

Based on phenotypic targeting, we hypothesised that connectivity mapping could be used to identify compounds or drugs that could shift undifferentiated DPCs towards an odontogenic phenotype. The aim of this study was to use publicly available gene expression datasets, together with ssCMap to identify compounds or drugs, already licensed for use in humans that could potentially induce osteo/odontogenic differentiation of DPCs and experimentally validate the effects in vitro. A subsidiary aim was to investigate the anti-inflammatory effect of identified compounds against cytokines associated with pulpitis (Rechenberg et al. 2016) using an ex vivo pulpitis model (Yonehiro et al. 2012, 2013).

\section{Methods}

Dataset selection

A search of the GEO database was performed using the keywords: 'dental pulp stem cells' 'DPCs,' 'differentiation,' 'lineage' 'osteogenesis', 'odontogenesis' 'osteogenic' and 'odontogenic.' Datasets were assessed for inclusion in the study using the criteria: cell type, induction of osteo/odontogenic differentiation, and the presence of both an undifferentiated control and differentiated treatment group.

Connectivity mapping

The Series Matrix Files (SMF) for the selected GEO datasets contained gene expression levels for all the probes used in the microarrays. These were downloaded and opened in Microsoft Excel (Microsoft Corporation, Redmond, WA, USA). Paired t-tests were performed on the gene expression levels measured in undifferentiated controls and cells that had undergone osteogenic/odontogenic differentiation. Genes with significant $(p<0.001)$ differential expression were selected and their fold change was calculated (Malcomson et al. 2016). Genes were given a score of +1 or -1 , depending on whether they were upregulated or downregulated.

The datasets used in this study were identified from GEO platform 6244 (GPL6244). To be compatible with ssCMap, the significantly differentially-expressed genes were converted to GPL96 probe IDs (See details in supplementary material). Connectivity mapping was carried out with the signature genes outlined in Table 1 using Zhang and Gant's ssCMap (Zhang \& Gant 2009) with default settings, which included a false connection tolerance of 1.0. Compounds with a significant positive connection score were considered for further analysis. The biological functions 
of the differentially expressed genes outlined in Table 1 were analysed using Ingenuity Pathway Analysis (IPA) software (Qiagen, Hilden, Germany).

Isolation and culture of DPCs

DPCs were derived by explant culture from immature permanent third molar teeth obtained in accordance with French ethics legislation. Cells were grown in minimal essential medium with Lglutamine supplemented with $10 \%$ foetal bovine serum (FBS), $100 \mathrm{Ul} / \mathrm{mL}$ penicillin and $100 \mu \mathrm{g} / \mathrm{mL}$ streptomycin and maintained in an incubator at $37 \mathrm{C}^{\circ}$ and $5 \% \mathrm{CO}_{2}$ throughout. DPCs were seeded in 6-well plates at a density of $3 \times 10^{4} / \mathrm{mL}$ and treatment protocols commenced when cells were 70 $80 \%$ confluent. Treatment protocols included, treatment with either $\alpha-M E M$ alone, osteogenic media (OM) containing $2 \mathrm{mM} \beta$-glycerophosphate (BGP) or $\alpha-M E M$ supplemented with the predicted compound.

MTT assay

DPCs were seeded in 96-well plates at a density of $3 \times 10^{4} / \mathrm{mL}$ and treated with normal media or predicted compound at concentrations ranging from $0.005 \mathrm{mM}$ to $10 \mathrm{mM}$ for 3,5 or 7 days. After the relevant time period $10 \mu \mathrm{L}$ MTT solution was added to each well for $2 \mathrm{~h}$ at $37^{\circ} \mathrm{C}$. Media was

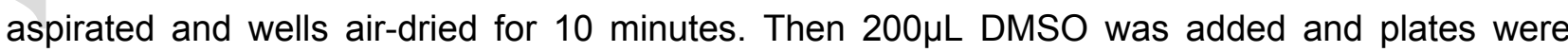
incubated at $37^{\circ} \mathrm{C}$ for a further 10 minutes. Samples were mixed and absorbance was measured at $510 \mathrm{~nm}$ using a Tecan GENios microplate reader.

Alkaline phosphatase (ALP) activity assay

ALP activity of control and treated cells was assessed at 14 days. Cell lysis was performed with $25 \mu \mathrm{L}$ radioimmunoprecipitation assay (RIPA) buffer on ice. An alkaline phosphatase diethanolamine detection Kit (Sigma Aldrich, St. Louis, Missouri, USA) was employed to perform the assay, as previously described (Winning et al. 2017). Briefly, the assay was a 96-well plate format, with $5 \mu \mathrm{L}$ of each cell lysate added to $240 \mu \mathrm{L}$ of reaction buffer. Following incubation for 20 minutes, the substrate p-nitrophenylphosphate (pNPP) was added ( $5 \mu \mathrm{L}$ of $0.67 \mathrm{M}$ pNPP solution). A negative control of $245 \mu \mathrm{L}$ reaction buffer with $5 \mu \mathrm{L}$ of $0.67 \mathrm{M}$ pNPP was also included. Absorbance was measured over 20 consecutive reads at $405 \mathrm{~nm}$.The ALP results were normalised to protein concentration determined using Micro BCA Protein Assay Kit (Thermo Fisher Scientific, Waltham, MA, USA).

\section{Alizarin red staining}

The mineralisation of treated and untreated cells was assessed with the Alizarin Red (AR) staining assay. Cells were fixed in ice cold $70 \%$ ethanol for 30 minutes at room temperature. Staining was performed with $1 \mathrm{~mL} 2 \%$ AR solution ( $\mathrm{pH}$ adjusted to $4.1-4.3$ using $\mathrm{NaOH}$ ) for 45 minutes at room temperature in the dark. Cells were destained with $1 \mathrm{~mL} 10 \%$ cetylpyridinium 
chloride in $10 \mathrm{mM}$ disodium hydrogen phosphate $(\mathrm{pH} 7)$ for 60 minutes. Absorbance was measured at $570 \mathrm{~nm}$.

Real-Time $q P C R$

RNA was extracted from treated and untreated cells using Qiagen RNeasy RNA Extraction Kit as per the manufacturer's instructions. cDNA synthesis was carried out with SuperScript VILO cDNA Synthesis Kit (Thermo Fisher Scientific) using 500 ng RNA. Real-time qPCR was carried out to quantify osteo/odontogenic genes ALPL, RUNX2, COL1A1, DSPP, DMP-1 and SPP1. A panel of genes identified by differential expression analysis namely: PELI 1, ERBB3, NARS and RABGAP1 were also analysed. GUSB and B2M were used as reference/housekeeping genes (Supp Table 1). qPCR was performed using a Stratgene Mx3005P (Agilent Technologies, Santa Clara, CA, USA) and data was recorded by MxPro software (Agilent Technologies). The thermal profile consisted of an initial period of 2 minutes at $50^{\circ} \mathrm{C}$ and 10 minutes at $95^{\circ} \mathrm{C}$, followed by 42 cycles of $30 \mathrm{~s}$ at $95^{\circ} \mathrm{C}$ and $1 \mathrm{~min}$ at $60^{\circ} \mathrm{C}$. For each primer a control with no template cDNA and a control with no reverse transcriptase were used.

Assessment of anti-inflammatory effect of the compound in an ex vivo pulpitis model

To assess the anti-inflammatory effect of the predicted compound, an ex vivo pulpitis model was established as previously described (Yonehiro et al. 2012, 2013). Briefly, DPCs and differentiated THP-1 cells were co-cultured in a 12-well transwell system (Corning Costar, Corning, NY, USA). THP-1 cells were seeded in the transwell upper chamber at the concentration of $1 \times 10^{5}$ cells per well. THP-1s were differentiated into macrophages by $24 \mathrm{~h}$, in the presence of $150 \mathrm{nM}$ phorbol 12-myristate 13-acetate (PMA, Sigma) followed by $24 \mathrm{~h}$ incubation in RPMI medium. DPCs were seeded in the lower chamber at a concentration of $2 \times 10^{5}$ cells per well. Cells were prepared in the transwells independently and just before the start of the experiments, both upper and lower chambers were combined as a co-culture, being separated only by a porous membrane $(0.4 \mathrm{~mm}$ pore diameter). To validate the co-culture system and to confirm previous findings that cytokines levels differ when cells are in monolayer compared to co-culture (Yonehiro et al. 2012), a set of experiments were performed in which differentiated THP-1 and DPCs in monolayer and coculture were treated with $1 \mu \mathrm{g} / \mathrm{mL}$ LPS for $24 \mathrm{~h}$ and the levels of the cytokines IL-8 and IL-B were measured in the cell supernatant using Duoset ELISA kits (R\&D Systems, Minneapolis ,MN, USA).

The co cultured THP-1 macrophages and DPCs were then treated with $1 \mu \mathrm{g} / \mathrm{mL}$ ultrapure E. coli LPS with or without the compound/drug of interest for $24 \mathrm{~h}$. Cytokines were measured from cell supernatants collected from both chambers of the co-culture system using a magnetic Luminex

This article is protected by copyright. All rights reserved 
assay kit (R\&D Systems) to simultaneously measure; IL-6, MMP-9, IL-8, CCL21, IL-1ß, IFN gamma, angiogenin, and angiopoietin.

To test the effect of the selected compound on the viability of differentiated THP-1 macrophages an MTT assay was performed as described above for DPCs, except that THP-1 cells were seeded at a density of $3 \times 10^{4}$ and treated with the predicted compound at a concentration of $0.05 \mathrm{mM}$ for $24 \mathrm{~h}$.

\section{Statistical analysis}

Data were summarised as means \pm standard deviations (SD) and analysed using GraphPad Prism software, version 5.0 (GraphPad Software, Inc., La Jolla, CA, USA). Means were compared using the $t$ - test or ANOVA followed by a Bonferroni post-test with the level of significance set at $p \leq 0.05$.

\section{Results}

Connectivity mapping

The GEO database search identified an appropriate dataset (GSE44677), which was available on the GPL6244 platform. Differential expression analysis and conversion of probe IDs to the GPL96 platform (as explained in supplementary material) resulted in the identification of 11 genes (Table 1) with a role in osteo/odontogenic differentiation. These were used as the gene expression signature to interrogate the connectivity mapping database. The ssCMap identified $0.1 \mathrm{mM}$ acetylsalicylic acid/aspirin (ASA) as the only drug with a significant positive connection score (Figure 1). The connection score for ASA was 3.583, the set score was 0.262 and the $p$ value was 0.0002 . ASA, which is non-steroidal anti-inflammatory drug was therefore, selected for laboratory analysis to determine if its predicted role in osteogenic/odontogenic induction could be confirmed in vitro. To understand the biological function of the differentially expressed genes shown in Table 1, a function and pathway analysis was performed using Ingenuity Pathway Analysis (IPA) software. Analysis revealed the most significantly perturbed biological functions in the dataset to be cellular processes associated with cell morphology, development, movement, growth and proliferation among others (Figure 2).

\section{Effect of ASA on cell viability}

The viability of DPCs was assessed following treatment with ASA $(0.005 \mathrm{mM}$ to $10 \mathrm{mM})$ using the MTT assay. At low concentrations $(<1 \mathrm{mM})$, which were suggested by the ssCMap, ASA did not affect the viability of DPCs or THP-1 cells, however, it was significantly affected at higher ASA concentrations (Figure 3. A, B, C \& Supp Figure 1).

ALP Activity and mineralisation assays 
The treatment of DPCs with either $0.05 \mathrm{mM}$ ASA or osteogenic media (OM) resulted in significantly higher $(p<0.001)$ ALP activity than untreated cells (Figure $3 \mathrm{D}$ ). Similarly, Alizarin red staining demonstrated higher mineralisation in ASA and OM treated cells compared to cells treated with $\alpha \mathrm{MEM}$ for 14 days, $p<0.001$ (Figure 3E\&F). There was no difference in the osteogenic effect between $0.05 \mathrm{mM}$ or $0.5 \mathrm{mM}$ ASA, and the lower concentration was used for the rest of the experiments as this approximately equates to the serum level of ASA after low dose 75 $\mathrm{mg}$ aspirin treatment (Patrignani et al. 2014).

qPCR and gene expression analysis

Treatment with $0.05 \mathrm{mM}$ ASA induced higher expression of the DMP1 gene compared to control culture after $24 \mathrm{~h}$. At day 7 only the DMP1 and DSPP genes were increased compared to control, however, expression of the osteogenic markers RUNX2, COL1A1, SPP1 and ALPL did not change significantly (Figure $3 \mathrm{G} \& \mathrm{H}$ ).

To verify the expression of the genes identified by differential expression analysis, four were selected for qPCR quantification. As shown in Supp Table 2, all the genes were expressed in DPCs and ASA treatment modulated their expression in a way that was relatively comparable to their fold change shown in Table 1.

Regulation of inflammatory cytokines with ASA

To assess if ASA at a concentration of $0.05 \mathrm{mM}$ exerted an anti-inflammatory effect, the level of a panel of cytokines previously reported as biomarkers for pulpitis (Rechenberg et al. 2016) was measured in an ex vivo pulpitis model using multiplex array. The production of cytokines in the co-culture pulpitis model was significantly higher than in THP-1 macrophages or DPCs grown as monolayers (Supp Figure 2). ASA treatment resulted in significant downregulation of the proinflammatory cytokines IL-6, MMP-9 and CCL21, but had no significant effect on expression of angiogenin, angiopoietin, IL-1 $\beta$, IFN gamma and IL-8 in the ex vivo pulpitis model (Figure 4).

\section{Discussion}

The main finding of this study was that connectivity mapping identified aspirin as a compound that could induce odontogenic differentiation. The gene expression signature of aspirin was shown by ssCMap to have the strongest correlation of any drug or compound in the reference database to the gene expression changes identified as occurring during osteo/odontogenic differentiation of DPCs. Aspirin is a prime candidate for drug repurposing due to its well-documented safety profile in humans. As such, the possible use of aspirin to repair damaged dentine in cases of deep caries and associated pulp inflammation could be particularly important. Connectivity mapping is a powerful approach that has principally been used to identify new applications for existing 
therapeutics, termed drug repurposing. The majority of previous CMap studies have focused on identifying whether an established drug, classically used to treat a certain disease or condition, shows promise in the treatment of an entirely unrelated disease or condition. For instance previously unrecognized analgesic and antinociceptive properties had been identified for the antihypertensive drug phenoxybenzamine (Chang et al. 2010). The results of the current study demonstrate that connectivity mapping can be used to identify compounds that can induce the differentiation of DPCs. In a similar recent study, parbendazole, primarily used as an anthelmintic agent, was identified as an inducer of osteogenic differentiation in bone marrow derived mesenchymal stem cells (Brum et al. 2015).

Support for the ability of aspirin to induce differentiation of stem cells comes from recent studies demonstrating osteogenic differentiation of DPCs (Yuan et al. 2018) and PDL stem cells (Abd Rahman et al. 2016). It was demonstrated in vitro and in vivo that aspirin, even at low concentrations, improved osteogenic differentiation of SHED via the (TERT)/Wnt/ $\beta$-catenin pathway (Liu et al. 2015). In these studies the use of aspirin was not underpinned by a rationale that supported its choice because it was superior to any other drug but rather they were exploratory studies of the effects of aspirin. In the current study the connectivity mapping approach identified aspirin as the highest scoring drug or compound matching the gene signature for osteo/odontgenic differentiation.

To validate the connectivity map findings, in vitro studies were performed to confirm the osteo/odontogenic effect of ASA. Upregulation of the odontogenic genes DMP-1 and DSPP occurred as well as evidence of mineralisation in response to a low concentration of $0.05 \mathrm{mM}$ ASA. The results, however, showed no changes in the expression of the known osteogenic genes RUNX2, SPP1 and COLA1. Similar findings have been reported in a study investigating the osteo/odontogenic differentiation of DPCs (Loison-Robert et al. 2018). DMP-1 and DSPP play important roles in odontoblast differentiation and dentine formation (D'Souza et al. 1997); however, their presence in bone and other calcified tissues makes it difficult to use them solely as odontoblast markers. The identification of the odontoblast-like cell phenotype remains challenging due to a lack of unique molecular or morphological markers (Duncan et al. 2019) and most studies use a panel of osteo/odontogenic genes as markers. Our results are in line with previous studies that demonstrated upregulation of DSPP and DMP-1 during odontoblast differentiation (He et al. 2008). An increase in SPP1 also occurred, but this did not reach statistical significance, however, since SPP1 is a late marker of osteo/odotogenic differentiation, its level may have increased beyond day 7 in the present study. RUNX2 is a master regulator of osteoblast differentiation and although its exact role in odontogenesis is not known studies have shown that downregulation of RUNX2 is a prerequisite for cells to proceed towards the odontoblast lineage 
(Gaikwad et al. 2001, Li et al. 2011, Widbiller et al. 2016). Therefore, the low levels of RUNX2 gene expression observed at 7 days may indicate odontogenic, rather than osteogenic differentiation of DPCs. Similarly, COL1A1 gene expression was previously reported to decrease with time during odontogenic differentiation of DPCs treated with Biodentine (Septodont, SaintMaur-des-Fossés, France) (Zanini et al. 2012, Widbiller et al. 2016) and the results at 24 hours and 7 days are in line with this trend.

The mechanism by which ASA induces osteo/odontogenic differentiation of DPCs is not known, but it is likely to be downstream of the genes that have been identified by our differential expression analysis. The IPA analysis showed these genes to have cellular functions related to development, growth and proliferation, and could therefore contribute to odontogenic differentiation of DPCs. The genes, RABGAP1 and PEL-I, are associated with autophagy (Popovic et al. 2012) and the NF-kB signalling pathway (Chang et al. 2009). These pathways have been shown to regulate odontogenic differentiation of DPCs. Autophagy has previously been shown to positively regulate odontogenic differentiation of DPCs (Pei et al. 2016, Yang et al. 2015), whereas NF-kB signalling has been reported to contribute either positively or negatively to this process (Feng et al. 2013, Hozhabri et al. 2015). NARS, which was also among the upregulated genes, is an important mediator of the effect of fibroblast growth factor on bone formation, by enhancing osteoblast survival and proliferation (Park et al. 2009). Among the genes identified as downregulated by the differential expression analysis was ERBB3, a receptor for neuregulin 1. This gene was previously shown to be downregulated during osteogenic differentiation of mesenchymal stem cells (MSCs) by Wnt3a, indicating cross talk between ERBB3 and Wnt3a in MSCs osteoblastic differentiation via Wnt/B Catenin signalling (Jullien et al. 2012). Thus, functions of some of the genes identified in our gene signature are relevant to osteo/odontogenic differentiation but further studies should explore their specific roles in DPCs.

A connectivity mapping approach to identify drugs that could be repurposed for the differentiation of stem cells appears promising, however, the process currently has limitations. There is a paucity of publicly available microarray data for differentiating cells, especially a niche cell type such as DPCs. Another disadvantage is that microarray probe IDs have to be converted to GPL96 to be compatible with ssCMap. This can result in the loss of probes and could affect the range of compounds identified by ssCMap. Nevertheless, the connectivity mapping discovery of aspirin although unexpected, is highly relevant to a proposed potential clinical application in pulp capping. Aspirin is a drug commonly used for the treatment of pain and inflammation and as an antiplatelet agent (Fuster \& Sweeny 2011). The mechanisms by which it produces these effects are varied, but are in part via inhibition of prostaglandin synthesis (Vane 1971) and of NF-KB signalling (Kopp \& Ghosh 1994). The anti-inflammatory effects of aspirin in these studies were 
observed at high therapeutic doses. The current study demonstrated that aspirin at a low concentration of $0.05 \mathrm{mM}$, which induced odontogenic differentiation, reduced the levels of inflammatory cytokines IL-6 and MMP-9 that are well documented biomarkers for pulpitis (Rechenberg et al. 2016, Zanini et al. 2017). The results also demonstrated that aspirin treatment has no effect on the angiogenic growth factors angiogenin and angiopoietin and the cytokines IL8 and IL-1 $\beta$. These findings demonstrate a selective anti-inflammatory effect of aspirin at low concentration that merit further investigation.

\section{Conclusion}

Connectivity mapping, a web based informatics method, was successfully used to identify aspirin as a candidate drug that could modulate the differentiation of DPCs. Aspirin was shown to induce odontogenic differentiation in DPCs in vitro and this together with its anti-inflammatory effects, make it a potential candidate for vital pulp therapies. Future work to investigate appropriate delivery methods for in vivo application is warranted.

\section{Acknowledgment}

Authors would like to thank Ms Catherine Fulton for her expert technical assistance. This work was funded by the School of Medicine Dentistry and Biomedical Sciences, Queen's University Belfast

\section{Conflict of Interest statement}

The authors have stated explicitly that there are no conflicts of interest in connection with this article. 


\section{References}

Abd Rahman F, Mohd Ali J, Abdullah M, Abu Kasim N H, Musa S (2016) Aspirin enhances osteogenic potential of periodontal ligament stem cells (PDLSCs) and modulates the expression profile of growth factor-associated genes in PDLSCs. Journal of Periodontology 87, 837-47. Brum AM, van Leeuwen JPT M, Eijken M et al. (2015) Connectivity Map-based discovery of parbendazole reveals targetable human osteogenic pathway. Proceedings of the National Academy of Sciences 112,12711-6.

Chang M, Jin W, Sun SC (2009) Peli1 facilitates TRIF-dependent Toll-like receptor signaling and proinflammatory cytokine production. Nature Immunology 10,1089-95.

Chang M, Smith S, Thorpe A, Barratt M J, Karim F (2010) Evaluation of phenoxybenzamine in the CFA model of pain following gene expression studies and connectivity mapping. Molecular Pain 6,56 .

D'Souza RN, Cavender A, Sunavala G, Alvarez J, Oshima T, Kulkarni AB, MacDougall M (1997) Gene expression of murine dentin matrix protein 1 (Dmp1) and dentin sialoprotein (DSPP) suggest distinct developmental functions in vivo. Journal of Bone and Mineral Research 12, 20409.

Duncan HF, Cooper PR, Smith AJ (2019) Dissecting dentine-pulp injury and wound healing responses: consequences for regenerative endodontics. International Endodontic Journal 52, 261-6.

Feng X, Feng G, Xing J et al. (2013) TNF- $\alpha$ triggers osteogenic differentiation of human dental pulp stem cells via the NF-kB signalling pathway. Cell Biology International 37,1267-75. Fuster V, Sweeny JM (2011) Aspirin: A historical and contemporary therapeutic overview. Circulation 123,768-78.

Gaikwad JS, Hoffmann M, Cavender AC, Bronckers AL, D'Souza RN ( 2001) Molecular insights into the lineage-specific determination of odontoblasts: the role of Cbfa1. Advances in Dental Research 15, 19- 24.

Goldberg M, Six N, Decup F (2001) Application of bioactive molecules in pulp-capping situations. Advances in Dental Research 15, 91-5.

$\mathrm{He} \mathrm{H}$, Yu J, Liu Y et al. (2008) Effects of FGF2 and TGFbeta1 on the differentiation of human dental pulp stem cells in vitro. Cell Biology International 32, 827-34.

Hozhabri NST, Benson MD, Vu, M D, et al. (2015) Decreasing NF-kB expression enhances odontoblastic differentiation and collagen expression in dental pulp stem cells exposed to inflammatory cytokines. PLOS ONE 10, e0113334.

Jullien N, Maudinet A, Leloutre B, Ringe J, Häupl T, Marie P J (2012) Downregulation of ErbB3 by Wnt3a contributes to wnt-induced osteoblast differentiation in mesenchymal cells. Journal of 
Cellular Biochemistry 113, 2047-56.

Kearney M, Cooper PR, Smith AJ, Duncan HF (2018) Epigenetic approaches to the treatment of dental pulp inflammation and repair: Opportunities and obstacles. Frontiers in Genetics 9, 311. Malcomson B, Wilson H, Veglia E, et al. (2016). Connectivity mapping (ssCMap) to predict A20inducing drugs and their antiinflammatory action in cystic fibrosis. Proceedings of the National Academy of Sciences 113, E3725-34.

Kopp E, Ghosh S (1994) Inhibition of NF-KB by sodium salicylate and aspirin. Science 265,956-9. Lamb J (2007) The Connectivity Map: A new tool for biomedical research. Nature Reviews Cancer 7, 54-60.

Lamb J, Crawford ED, Peck, D et al. (2006) The Connectivity Map : Using gene expression signatures to connect small molecules, genes and disease. Science 313, 1929-35.

Li S, Kong H, Yao N, et al. (2011) The role of runt-related transcription factor 2 (Runx2) in the late stage of odontoblast differentiation and dentin formation. Biochemistry \& Biophysics Research Communication 410, 698-704.

Li Z, Cao L, Fan M, Xu Q (2015) Direct pulp capping with calcium hydroxide or mineral trioxide aggregate: A meta-analysis. Journal of Endodontics 41,1412-7.

Liu Y, Chen C, Liu S et al. (2015) Acetylsalicylic acid treatment improves differentiation and immunomodulation of SHED. Journal of Dental Research 94,209-18.

Loison-Robert, LS, Tassin M, Bonte E et al. (2018) In vitro effects of two silicate-based materials, Biodentine and BioRoot RCS, on dental pulp stem cells in models of reactionary and reparative dentinogenesis. PLoS ONE 13, e0190014.

Murray P E, García-Godoy F (2006) The incidence of pulp healing defects with direct capping materials. American Journal of Dentistry 19,171-7.

Musa A, Ghoraie LS, Zhang SD et al. (2017) A review of connectivity map and computational approaches in pharmacogenomics. Briefings in Bioinformatics 18,903.

Neves V C M, Babb R, Chandrasekaran D, Sharpe PT (2017) Promotion of natural tooth repair by small molecule GSK3 antagonists. Scientific Reports 7,39654

Park S J, Kim S H, Choi H S, Rhee Y, Lim S K (2009) Fibroblast growth factor 2-induced cytoplasmic asparaginyl-tRNA synthetase promotes survival of osteoblasts by regulating antiapoptotic PI3K/Akt signaling. Bone 45,994-1003.

Patrignani P, Tacconelli S, Piazuelo E et al. (2014) Reappraisal of the clinical pharmacology of low-dose aspirin by comparing novel direct and traditional indirect biomarkers of drug action. Journal of Thrombosis and Haemostasis 12,1320-30.

Pei F, Wang HS, Chen Z, Zhang L (2016) Autophagy regulates odontoblast differentiation by suppressing NF-kB activation in an inflammatory environment. Cell Death \& Disease 7,e2122. 
Phung S, Lee C, Hong C (2017) Effects of bioactive compounds on odontogenic differentiation and mineralization. Journal of Dental Research 96,107-115.

Popovic D, Akutsu M, Novak I, Harper J W, Behrends C, Dikic I (2012) Rab GTPase-activating proteins in autophagy: Regulation of endocytic and autophagy pathways bydirect binding to human ATG8 modifiers. Molecular and Cellular Biology 32,1733-44.

Ramsey JM, Kettyle LMJ, Sharpe DJ et al. (2013) Entinostat prevents leukemia maintenance in a collaborating oncogene-dependent model of cytogenetically normal acute myeloid leukemia. Stem Cells 31,1434-45.

Rechenberg DK,Galicia JC, Peters OA (2016) Biological markers for pulpal inflammation: A systematic review. PLOS ONE 29, 11 (11):e0167289.

Shrestha S, Kishen A (2017) Bioactive Molecule Delivery Systems for Dentin-pulp Tissue Engineering. Journal of Endodontics 43,733-44.

Sloan AJ, Smith AJ (2007) Stem cells and the dental pulp: Potential roles in dentine regeneration and repair. Oral Diseases 13,151-7.

Vane JR (1971) Inhibition of prostaglandin synthesis as a mechanism of action for aspirin-like drugs. Nature New Biology 231, 232-5.

Widbiller M, Lindner SR, Buchalla W et al. (2016) Three-dimensional culture of dental pulp stem cells in direct contact to tricalcium silicate cements. Clinical Oral Investigations 20, 237- 46. Winning L, Robinson L, Boyd AR, EI Karim IA, Lundy FT, Meenan BJ (2017) Osteoblastic differentiation of periodontal ligament stem cells on non-stoichiometric calcium phosphate and titanium surfaces. Journal of Biomedical Materials Research - Part A 105,1692-1702.

Yang JW, Zhang YF, Wan CY et al. (2015) Autophagy in SDF-1a-mediated DPSC migration and pulp regeneration. Biomaterials 44,11-23.

Yonehiro J, Yamashita A, Yoshida Y, et al. (2012) Establishment of an ex vivo pulpitis model by co-culturing immortalized dental pulp cells and macrophage. International Endodontic Journal 45, 1103-8.

Yonehiro J, Yoshida Y, Yamashita A, et al. (2013) Flavonol-containing phosphorylated pullulan may attenuate pulp inflammation. International Endodontic Journal 46, 119-27.

Yuan M, Zhan Y, Hu W et al. (2018) Aspirin promotes osteogenic differentiation of human dental pulp stem cells. International Journal of Molecular Medicine 42,1967-76.

Zanini M, Sautier JM, Berdal A et al. (2012) Biodentine induces imortalised murine pulp cell differentiation into odontoblast-like cells and stimulates biomineralisation. Journal of Endodontics 38, $1220-1226$.

Zanini M, Meyer E, Simon S (2017) Pulp inflammation diagnosis from clinical to inflammatory mediators: A systematic review. Journal of Endodontics 43, 1033-1051 
Zhang SD, Gant TW (2008) A simple and robust method for connecting small-molecule drugs using gene-expression signatures. BMC Bioinformatics 9, 258.

Zhang S D, Gant T W (2009) ssCMap: An extensible Java application for connecting smallmolecule drugs using gene-expression signatures. BMC Bioinformatics 89,1110-3. 


\section{Figure Legends}

Figure 1 The sscMap output for the signature genes outlined in Table 1. The map is a plot of the distribution of candidate compounds that may enhance (right side) or suppress (left side) the desired effect (osteo/odontogenesis). Significant candidates are above the green line with either enhancing (right hand side) or reducing (left hand side) effects. The green line is a predetermined threshold $p$-value calculated using $1 / N$ where $N$ is the number of reference datasets. In this case $N$ is 3738 resulting in a $p$ value of 0.0003 , at which the green line is set. ASA corresponds to the dot above the green line (significant) on the right side (enhancer). The connection score for ASA was 3.583, the set score was 0.262 and the p value was 0.0002 .

Figure 2 Graph showing molecular and cellular function analysis of the differentially expressed genes using Ingenuity Pathway Analysis (IPA). The minimum significance level scored as -log ( $p$ value) was calculated with Fisher's exact test. The significance value associated with a function in global analysis is a measure for how likely it is that genes participate in that function.

Figure 3 Effect of ASA on DPCs viability and osteo/odontogenic differentiation: (A-C) MTT graphs showing that ASA has no effects on DPC viability at concentrations $<1 \mathrm{mM}$ during culture for 3 (A), 5 (B) and 7 (C) days. (D) DPCs treated with 0.05mM ASA or osteogenic media (OM) for 14 days showed high ALP activity compared to non-treated cells. (E\&F) DPCs showed evidence of mineralization in response to ASA and OM, which, was significantly higher than untreated cells. Expression of osteo/odontogenic genes after exposure to $0.05 \mathrm{mM}$ ASA quantified by qPCR showed: (G) DMP1 gene expression increased after 24 hours; $(H)$ Both DMP1 and DSPP gene expression increased after 7 days. Data represent mean+/- SD; ${ }^{* *} p<0.001,{ }^{* *} p<0.01$, * $\mathrm{p}<0.05$, as determined by ANOVA with Bonferroni post hoc correction. Results represent an average of three independent experiments.

Figure 4 Effect of ASA treatment on cytokine release in ex vivo pulpitis model: pro-inflammatory cytokines IL-6, CCL21 and MMP-9 were significantly reduced in response to treatment with $0.05 \mathrm{mM}$ ASA, while expression of the angiogenic factors angiogenin and angiopoietin 1 and cytokines, INF gamma, IL-I $\beta$, IL-8 was unchanged. Data represent mean+/- SD; ${ }^{* * *} p<0.001,{ }^{*} p$ $<0.05$ as determined by ANOVA-test with Bonferroni post hoc correction. Results represent an average of three independent experiments.

This article is protected by copyright. All rights reserved 
Table 1 DPCs osteo/odontogenic gene signature List of genes (1) upregulated and (-1) downregulated identified by differential expression analysis. Only these 11 genes were ssCMAp compatible and therefore were used as the gene signature.

\begin{tabular}{|c|c|c|c|c|c|c|}
\hline GPL96_ID & Gene Symbol & Gene Title & Fold change & Fold Value & P Value & Conversion \\
\hline 204028_s_at & RABGAP1 & RAB GTPase activating protein 1 & 1.0507 & 0.071418426 & 0.00014075 & +1 \\
\hline 218319_at & PELI1 & Pellino homolog 1 (Drosophila) & 1.173 & 0.23031762 & 0.000262363 & +1 \\
\hline 218040_at & PRPF38B & PRP38 pre-mRNA processing factor 38 (yeast) domain containing B & 1.066 & 0.092466009 & 0.000326306 & +1 \\
\hline 219808_at & SCLY & selenocysteine lyase & 1.051 & 0.071873327 & 0.000422738 & +1 \\
\hline 200027_at & NARS & asparaginyl-tRNA synthetase & 1.044 & 0.062898243 & 0.000903409 & +1 \\
\hline 220359_s_at & ARPP21 & cAMP-regulated phosphoprotein, 21kDa & 0.965 & -0.051191504 & 0.000109973 & -1 \\
\hline 207399_at & BFSP2 & beaded filament structural protein 2, phakinin & 0.862 & -0.214114885 & 0.000396292 & -1 \\
\hline 208415_x_at & ING1 & inhibitor of growth family, member 1 & 0.915 & -0.127681727 & 0.000494042 & -1 \\
\hline 202454_s_at & ERBB3 & v-erb-b2 erythroblastic leukaemia viral oncogene homolog 3 (avian) & 0.9284 & -0.1017104776 & 0.00059818 & -1 \\
\hline 204416_x_at & APOC1 & apolipoprotein C-I & 0.9425 & -0.085298083 & 0.00089721 & -1 \\
\hline
\end{tabular}


Figure 1

SSC plot: $Y=-\log 10$ (pvalue)

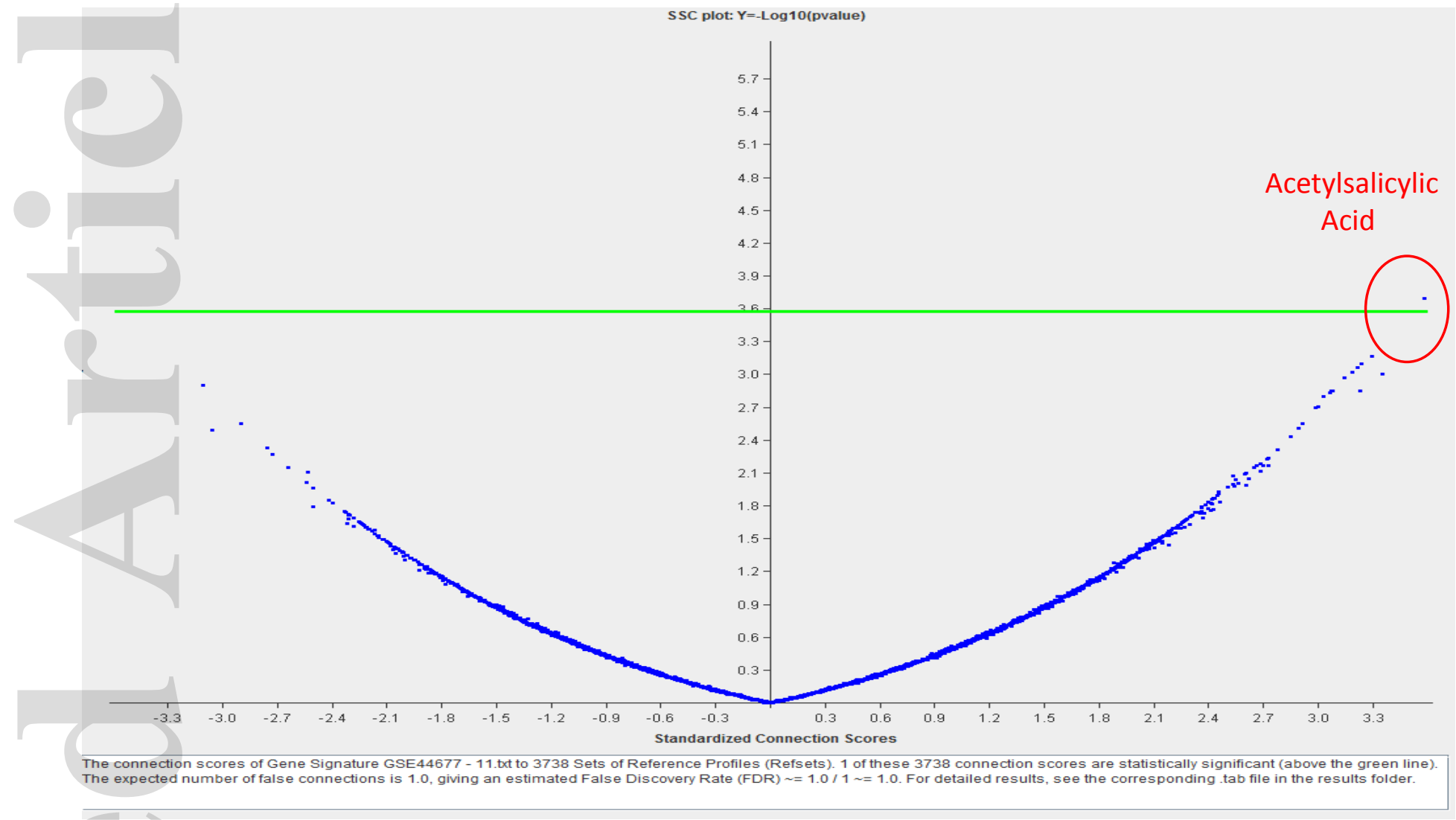

This article is protected by copyright. All rights reserved 


\section{Figure 2}

Cell Morphology

Cell-To-Cell Signaling and Interaction

Embryonic Development

Endocrine System Development and Function

Lipid Metabolism

Nervous System Development and Function

Organ Morphology

Organismal Development

Small Molecule Biochemistry

Tissue Development

Cell cycle

Cellular Development

Cellular Growth and Proliferation

Cellular Movement

Cellular Response to Therapeutics

Cellular Function and Maintenance

Molecular Transport

Cell Death and Survival

Cellular Assembly and Organization

Inflammatory Response

Carbohydrate Metabolism

Cell-mediated Immune Response

Hematological System Development and Function

Immune Cell Trafficking

Organ Development

Drug Metabolism

Cardiovascular System Development and Function Connective Tissue Development and Function Cell Signaling

Post-Translational Modification

Protein Synthesis

Skeletal and Muscular System Development and Function DNA Replication, Recombination, and Repair

Inflammatory Disease

Nucleic Acid Metabolism

Protein Trafficking

2000-2018 OIAGEN. All rights reserved.

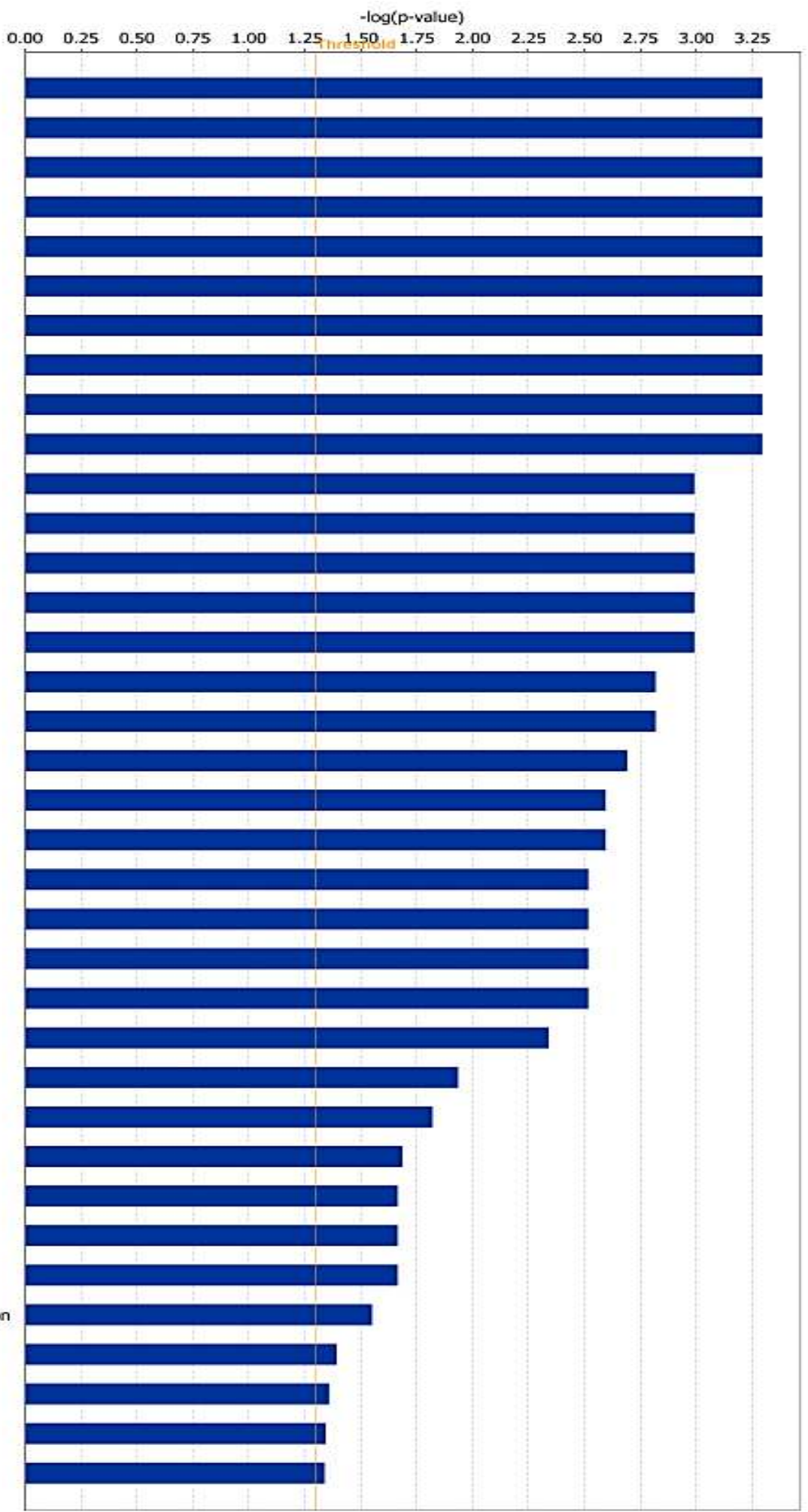

This article is protected by copyright. All rights reserved 


\section{Figure 3}

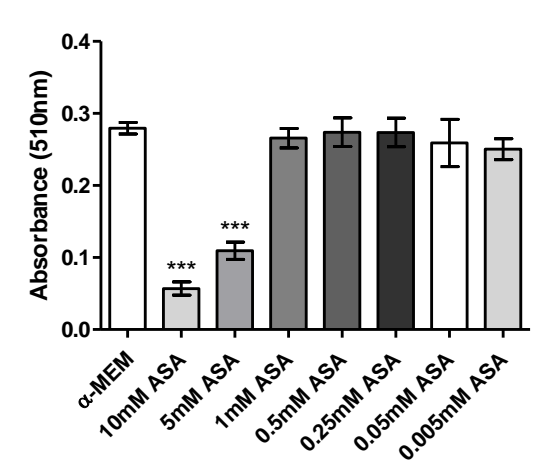

D

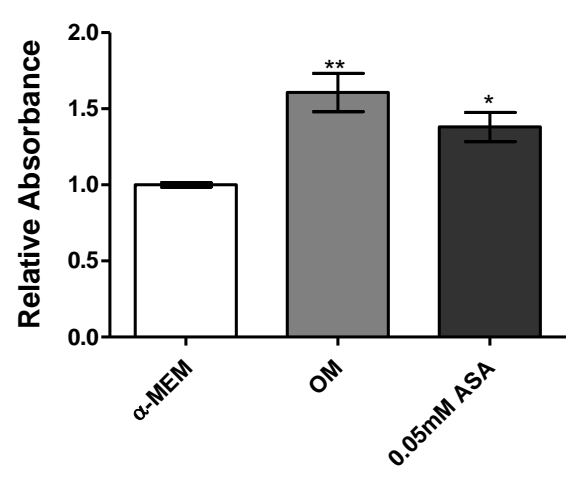

G

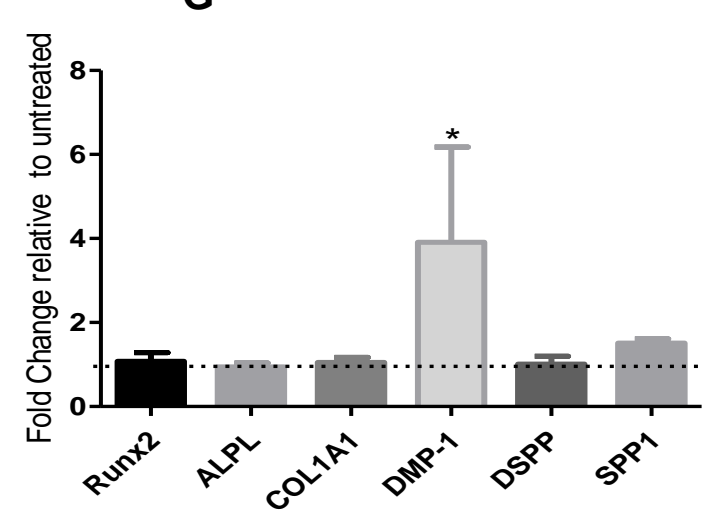

B

C

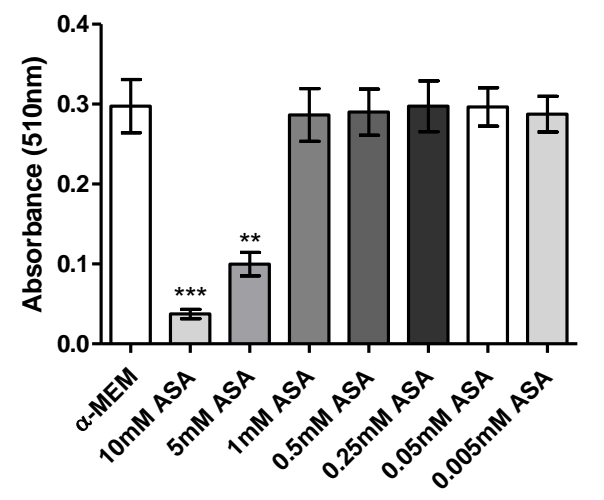

F

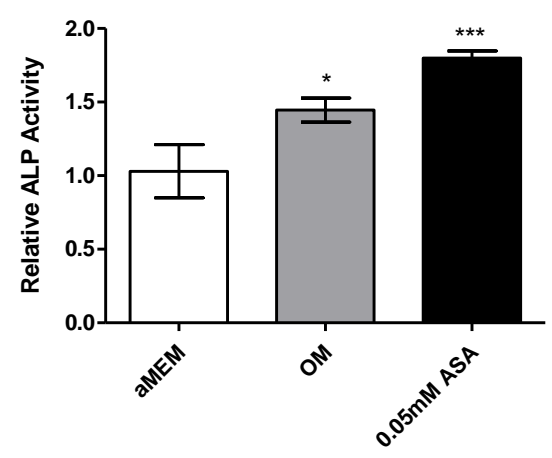

H

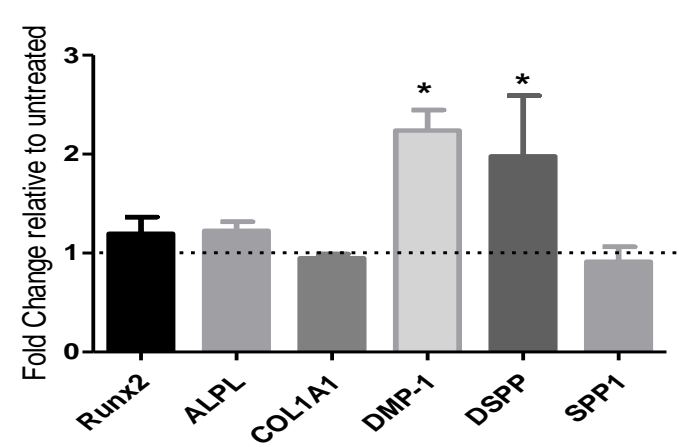


Figure 4

CTRL

$\square$ LPS

LPS+ASA
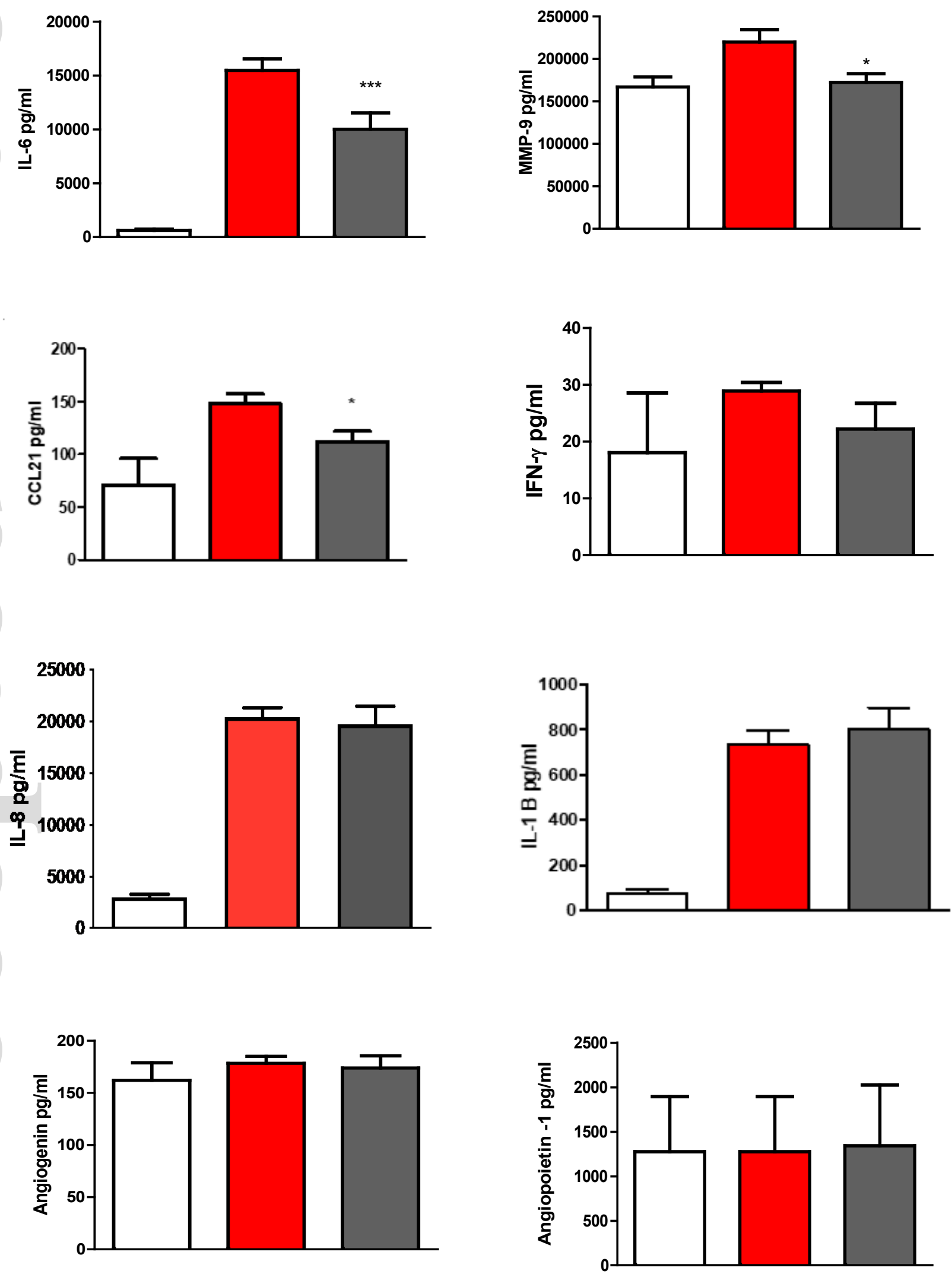

This article is protected by copyright. All rights reserved 